European Psychiatry

www.cambridge.org/epa

\section{Research Article}

Cite this article: Jaen-Moreno MJ, Feu N, del Pozo GI, Gómez C, Carrión L, Chauca GM, Guler I, Montiel FJ, Sánchez MD, Alcalá JA Gutierrez-Rojas L, Molina V, Bobes J, Balanzá-Martínez V, Ruiz-Rull C, Sarramea F (2021). Chronic obstructive pulmonary disease in severe mental illness: A timely diagnosis to advance the process of quitting smoking. European Psychiatry, 64(1), e22, 1-11 https://doi.org/10.1192/j.eurpsy.2021.12

Received: 28 October 2020

Revised: 12 January 2021

Accepted: 09 February 2021

\section{Keywords:}

bipolar disorder; COPD; early detection; schizophrenia; smoking

\section{Author for correspondence:}

*Vicent Balanzá Martínez,

E-mail: vicente.balanza@uv.es

\title{
Chronic obstructive pulmonary disease in severe mental illness: A timely diagnosis to advance the process of quitting smoking
}

\author{
M. J. Jaen-Moreno ${ }^{1,2}$ (1), N. Feu ${ }^{3}$, G. I. del Pozo ${ }^{1,4}$, C. Gómez ${ }^{1,5}$, L. Carrión ${ }^{1,6}$, \\ G. M. Chauca ${ }^{1,6}$, I. Guler ${ }^{7}$, F. J. Montiel ${ }^{1,5}$, M.D. Sánchez ${ }^{5}$, J. A. Alcalá1,4 (D, \\ L. Gutierrez-Rojas ${ }^{8}$ (D) , V. Molina ${ }^{9,10,11,12}$, J. Bobes $^{13}$ (D) , V. Balanzá-Martínez ${ }^{14 \star}$ (D), \\ C. Ruiz-Rull ${ }^{1,15}$ and F. Sarramea ${ }^{1,2,4,16}$
}

${ }^{1}$ Instituto Maimónides de Investigación Biomédica de Córdoba (IMIBIC), Córdoba, Spain; ${ }^{2}$ Departamento de Ciencias Morfológicas y Sociosanitarias, Universidad de Córdoba, Córdoba, Spain; ${ }^{3}$ Unidad de Gestión Clínica de Neumología, Hospital Universitario Reina Sofía, Córdoba, Spain; ${ }^{4}$ Unidad de Gestión Clínica de Salud Mental, Hospital Universitario Reina Sofía, Córdoba, Spain; ${ }^{5}$ Unidad de Gestión Clínica de Salud Mental, Complejo Hospitalario de Jaén, Jaén Spain; ${ }^{6}$ Unidad de Gestión Clínica de Salud Mental, Hospital Infanta Margarita, Cabra, Spain; ${ }^{7}$ Instituto Maimónides de Investigación Biomédica de Córdoba (IMIBIC), Área de gestión de la investigación, Córdoba, Spain; ${ }^{8}$ Grupo de Investigación Psiquiatría y Neurociencias (CTS-549), Instituto de Neurociencias, Universidad de Granada, Granada, Spain; ${ }^{9}$ Psychiatry Department, School of Medicine, University of Valladolid, Valladolid, Spain; Psychiatry Service, Clinical Hospital of Valladolid, Valladolid, Spain; ${ }^{10}$ Neurosciences Institute of Castilla y Leon (INCYL), University of Salamanca, Salamanca, Spain; Instituto de Investigacion Biomedica de Salamanca (IBSAL), Salamanca, Spain; ${ }^{11}$ CIBERSAM (Biomedical Research Network in Mental Health), Instituto de Salud Carlos III, Madrid, Spain; ${ }^{12}$ Instituto de Investigación Biomédica de Salamanca (IBSAL), Salamanca, Spain; ${ }^{13}$ Faculty of Medicine and Health Sciences-Psychiatry, Universidad de Oviedo, ISPA, INEUROPA, CIBERSAM, Oviedo, Spain; ${ }^{14}$ Unitat Docent de Psiquiatría i Psicología Médica, Departament de Medicina, Universitat de València, CIBERSAM, Valencia, Spain; ${ }^{15}$ Unidad de Gestión Clínica de Medicina Familiar y Comunitaria, Hospital Universitario Reina Sofía, Córdoba, Spain and ${ }^{16}$ Centro de Investigación Biomédica en Red de Salud Mental, CIBERSAM, Oviedo, Spain

\begin{abstract}
Background. This study has two main objectives: to describe the prevalence of undetected chronic obstructive pulmonary disease (COPD) in a clinical sample of smokers with severe mental illness (SMI), and to assess the value of the Tobacco Intensive Motivational Estimated Risk tool, which informs smokers of their respiratory risk and uses brief text messages to reinforce intervention.

Method. A multicenter, randomized, open-label, and active-controlled clinical trial, with a 12-month follow-up. Outpatients with schizophrenia (SZ) and bipolar disorder were randomized either to the experimental group-studied by spirometry and informed of their calculated lung age and degree of obstruction (if any) - or to the active control group, who followed the 5 A's intervention.

Results. The study sample consisted of 160 patients $(71.9 \% \mathrm{SZ}), 78.1 \%$ of whom completed the 12-month follow-up. Of the patients who completed the spirometry test, $23.9 \%$ showed evidence of COPD (77.8\% in moderate or severe stages). TIMER was associated with a significant reduction in tobacco use at week 12 and in the long term, $21.9 \%$ of patients reduced consumption and $14.6 \%$ at least halved it. At week 48 , six patients (7.3\%) allocated to the experimental group achieved the seven-day smoking abstinence confirmed by CO (primary outcome in terms of efficacy), compared to three (3.8\%) in the control group.

Conclusion. In this clinical pilot trial, one in four outpatients with an SMI who smoked had undiagnosed COPD. An intensive intervention tool favors the early detection of COPD and maintains its efficacy to quit smoking, compared with the standard 5 A's intervention.
\end{abstract}

(c) The Author(s), 2021. Published by Cambrid University Press on behalf of the European Psychiatric Association. This is an Open Access article, distributed under the terms of the Creative Commons AttributionNonCommercial-NoDerivatives licence (http:// creativecommons.org/licenses/by-nc-nd/4.0/), which permits non-commercial re-use, distribution, and reproduction in any medium, provided the original work is unaltered and is properly cited. The written permission of Cambridge University Press must be obtained for commercial reuse or in order to create a derivative work. 
far been based on surveys and population studies. Therefore, very little evidence on the onset of lung damage and the possibilities of early diagnosis in SMI is available [13].

Safe and effective pharmacological approaches exist for treating smoking in SMI $[14,15]$. Persuading as many patients as possible to undergo treatment remains as one of the key challenges [16]. The preparation phase, which boosts motivation and schedules the new attempt, is an essential step [17]. Nowadays, more personalized interventions with specific designs are recommended to achieve that goal [18].

New tools, such as using personalized information and mobile technology, have proved effective in promoting health and generating healthy behavior [19]. In smoking treatments in the general population, informing the subject about their individual risks using lung scans, genetic tests, or spirometry, encourages new attempts to quit, helps to reduce consumption, and can increase cessation rates [20-25]. In patients with SZ or BD, who are usually less aware of the risks for their health [26,27], the efficacy of giving personalized information about risk and prevention has not been fully explored. Even the most basic feature of mobile technology, the short message service (SMS), can reach devices everywhere and has been shown to generate and maintain motivation to quit smoking [28].

Patients with SMI have less access to preventive health care [29] as well as a pressing need to advance toward smoking cessation. The Tobacco Intensive Motivational Estimated Risk tool (TIMER) is based on the structure of the $5 \mathrm{~A}$ 's, which is the standard guidelines for intervention in clinical practice (Ask, Advise, Assess, Assist, and Arrange) [22]. Here, it reports spirometry results, the Gold Standard in the study of lung function [30], to inform the patient of their respiratory risk and it keeps the message going via SMS technology.

This is a clinical pilot trial with two main objectives: (a) to evaluate previously undiagnosed lung damage in a sample of smokers with SZ and BD; and (b) to analyze the effectiveness of the TIMER tool to help smoking cessation, reduce consumption, and generate attempts to quit, compared to the $5 \mathrm{~A}$ 's reference intervention. It could present an opportunity to promote the early diagnosis of COPD in the clinical context of community care, without losing its value as a way of activating the quitting process.

\section{Methods}

\section{Study design and settings}

This was a multicenter, randomized, active-controlled, open-label, pilot trial with a 12-month follow-up. The study was carried out in nine mental health centers (MHC) in urban and rural community settings in four provinces of Andalusia-Córdoba, Jaén, Málaga, and Granada-in southern Spain. The study protocol (11/2016) was approved by the Research and Ethics Committee of the Reina Sofía University Hospital (HURS) in Córdoba. All the participants gave their written informed consent before randomization. The ClinicalTrials.gov identifier was NTC03583203.

\section{Sample recruitment and randomization}

Over a 6-month period, all consecutive outpatients at the MHCs who met the selection criteria were invited to participate in the study.

\section{Inclusion criteria}

1. Aged between 40 and 70 years.
2. Patients diagnosed with $\mathrm{SZ}$ or $\mathrm{BD}$, according to the Diagnostic and Statistical Manual of Mental Disorders, 4th edition, text revision (DSM-IV-TR) [31].

3. Active smokers (current consumption of at least 10 cigarettes per day (CPD) with a cumulative consumption of at least 10 packs/year.

4. Clinical stability, which was psychometrically confirmed for participants with BD ([Hamilton Rating Scale for Depression [32] total score lower than 14 and Young Mania Rating Scale [33] total score lower than 6]) and SZ ([Positive and Negative Syndrome Scale [34] total score lower than 70]).

\section{Exclusion criteria}

1. Patients with a current or previous diagnosis of respiratory conditions.

2. Existence of any pathology which makes it inadvisable to perform spirometry.

3. Patients who, due to their intellectual disability or other psychiatric disorder, do not understand study procedures or cannot adequately perform spirometry.

The randomization was carried out using the Redcap program [35], as implemented by the Technological and Bioinformatic Innovation Unit at the IMIBIC (Maimónides Institute of Biomedical Research) in Córdoba, Spain. Randomization was centralized, performed in permuted blocks, and stratified by MHC, age, gender, and pathology.

\section{Intervention}

The experimental and control interventions were performed by the same psychiatrists and mental health nurses in charge of patients' routine follow-ups at each MHC. They received additional training from experts from the addiction treatment network and specialists in functional tests from the Pneumology Service at the HURS, in Córdoba.

In the experimental intervention, personalized information about the lung damage and prevention opportunities were included, which allowed patients to compare their lung age with chronological age. Moreover, patients received information regarding the evidence of undetected COPD (or lack thereof) and its staging. For further details, please see the supplementary material.

\section{Outcomes}

\section{Lung damage}

1. Primary outcome: the presence of COPD confirmed after bronchodilation (Forced Expiratory Volume in first second $($ FEV1)/forced vital capacity $(\mathrm{FVC})<0.7)$ and COPD staging following the Global Initiative for Chronic Obstructive Lung Disease [36] - 1(mild): FEV1 > 80\%; 2 (moderate): 50\% < FEV1 $<80 \%$; 3 (severe): $30 \%<$ FEV1 < 50\%; 4 (life-threatening): FEV1 $<30 \%$ - and the combined classification-lung function and respiratory clinical condition-for COPD (see Table 4).

2. Secondary outcome: percentage of FEV1 compared with expected level, based on the Fletcher and Peto model [37]; and the presence of restrictive pattern $(\mathrm{FVC}<80 \%$ and $\mathrm{FEV} 1 /$ FVC $>70 \%)$.

\section{Efficacy}

1. Primary outcome: smoking cessation at 48 weeks from the intervention (self-reported abstinence for the past 7 days, 


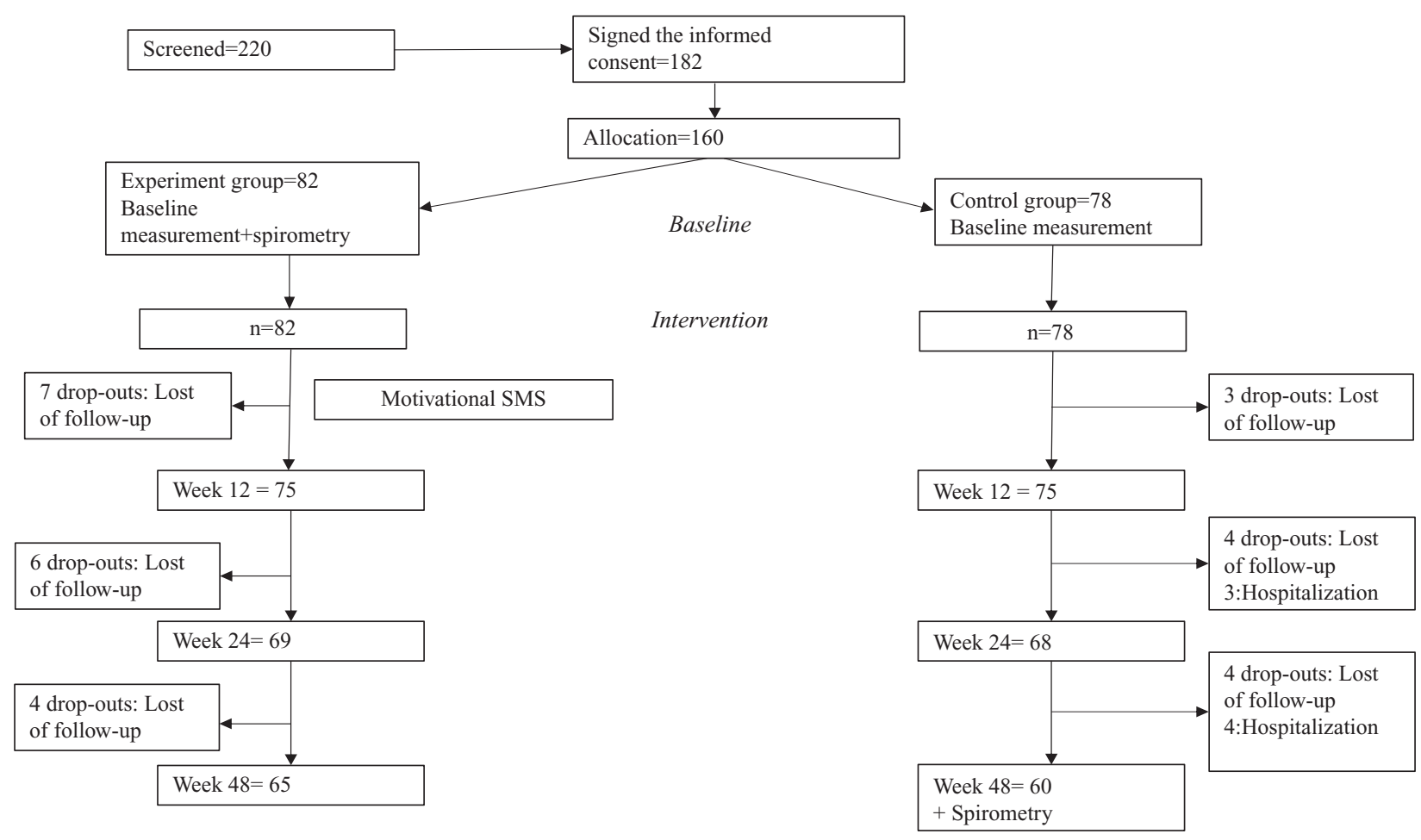

Figure 1. Flow diagram in the study design. Patient disposition.

confirmed by CO-oximetry with expired carbon monoxide $(\mathrm{CO})<10$ particles per million (ppm)).

2. Secondary outcomes: (a) smoking cessation at 3 and 6 months; (b) reduction in the tobacco consumed, as self-reported by the patient and confirmed by expired $\mathrm{CO}$ at 12,24 , and 48 weeks; and (c) number of quit attempts - at least $24 \mathrm{~h}$ smoking-freeover the follow-up.

\section{Follow-up and assessments}

Subjects from the two study arms were evaluated four times. The first visit (baseline) was scheduled before the intervention, and follow-up visits at 12,24, and 48 weeks after the intervention (see Figure 1). In addition, all patients attended regularly scheduled appointments with their psychiatrists as part of their usual clinical follow-up.

The sociodemographic and clinical variables, smoking habit data, motivation levels, number of attempts to quit, history of other substance use, medical comorbidities, and anthropometric measurements were collected, as specified in the scheduled task in the supplementary material. For further details, please see the previously published protocol [38].

The descriptive variables of the lung damage study-volumes and clinical respiratory observations-were obtained from the two clinical groups: (a) the subjects in the intervention arm at the baseline visit (here the data were used as personalized information on lung risk and as a basis for the assessed intervention); (b) those patients in the control arm who had finished the follow-up and were interested in learning about their lung function. The results describing lung damage are the summary of the data from these two groups.

\section{Statistical analysis}

Continuous variables are described as mean and standard deviations values, the categorical variables as frequencies and proportions. For the sample description, the Mann-Whitney and Chi-square tests were used to compare continuous variables between the groups and over time, as appropriate. The Last Observation Carried Forward method was used as a denominator of proportions, which refers to the total number of randomized patients.

A linear mixed-effects model was performed to evaluate the changes in the short-term reduction in smoking (from baseline to 12 weeks) and in the long-term reduction in smoking (from baseline to 48 weeks) with time, socio-demographics, smoking habit, and clinical data taken as fixed effects, and a random intercept and random slope effects. The variables which are clinically considered to exert a possible effect on reduction in smoking were introduced into a variable selection in a multivariate model, comparing the nested models using the log-likelihood values.

The linear mixed-effects models deal with missing values and measurement error taking into account the between- and withinsubject variability. The variance-covariance structure was fixed to an unstructured matrix and the random effects and error terms were assumed to have a normal distribution.

For the primary goal of efficacy and estimating abstinence at 12 months of 3 and $15 \%$ in the control and experimental arms, a sample size calculation of 86 patients per arm was made, with an added $20 \%$ for possible losses during follow-up (for more details see the published protocol) [38]. To explore the possible risk factors for the obstructive pattern-age, sex, psychiatric diagnosis, economic status, size of city of residence, level of smoking dependence, combined consumption of tobacco and cannabis, cohabitation with other smokers, antipsychotic treatment, abdominal circumference, body mass index, diabetes, and dyslipidemia - and due to the small sample size (for having a greater number of parameters than the sample), we performed just a univariate logistic regression for all possible characteristics of patients. All the analyses were performed using $\mathrm{R}$ software (version 3.6.2) and $p$-values $<0.05$ were 
considered as statistically significant. The results are presented as Odds Ratios and 95\% CI only for the statistically significant variables.

\section{Results}

A total of 220 patients attending their scheduled appointments were informed about the study procedures. Of them, 182 patients met all the criteria for inclusion and none for exclusion, and signed the informed consent. Given that 22 patients withdrew their consent before enrolment, 160 patients were finally randomized. The final sample size was somewhat below that calculated at the beginning. Taking as reference the estimated abstinence rates at 12 months of 3 and $15 \%$ in the control and experimental arms, respectively, the post-hoc statistical power of this study was $77 \%$ with $95 \%$ confidence intervals.

The final sample of the present study was made up of 160 patients, who were randomized to the EG $(n=82)$ and the CG $(n=78)$. Of the total sample, in $115(71.9 \%)$ diagnosis was SZ and $45(28.1 \%)$ was BD. The dropout rate was $21.87 \%$ : seven cases (4.37\%) due to hospital admission after a relapse, and the remaining $28(17.5 \%)$ who missed their appointments (Figure 1 shows the patients' disposition).

The demographic, clinical, anthropometric, and smoking characteristics at baseline are shown in Table 1 . There were no significant differences between groups. Two patients in the CG (Nicotine replacement therapy (TSN)) and two in the EG (1 TSN and 1 Varenicline) were under pharmacological treatment.

Table 1. Sociodemographic, clinical, and smoking characteristics at baseline.

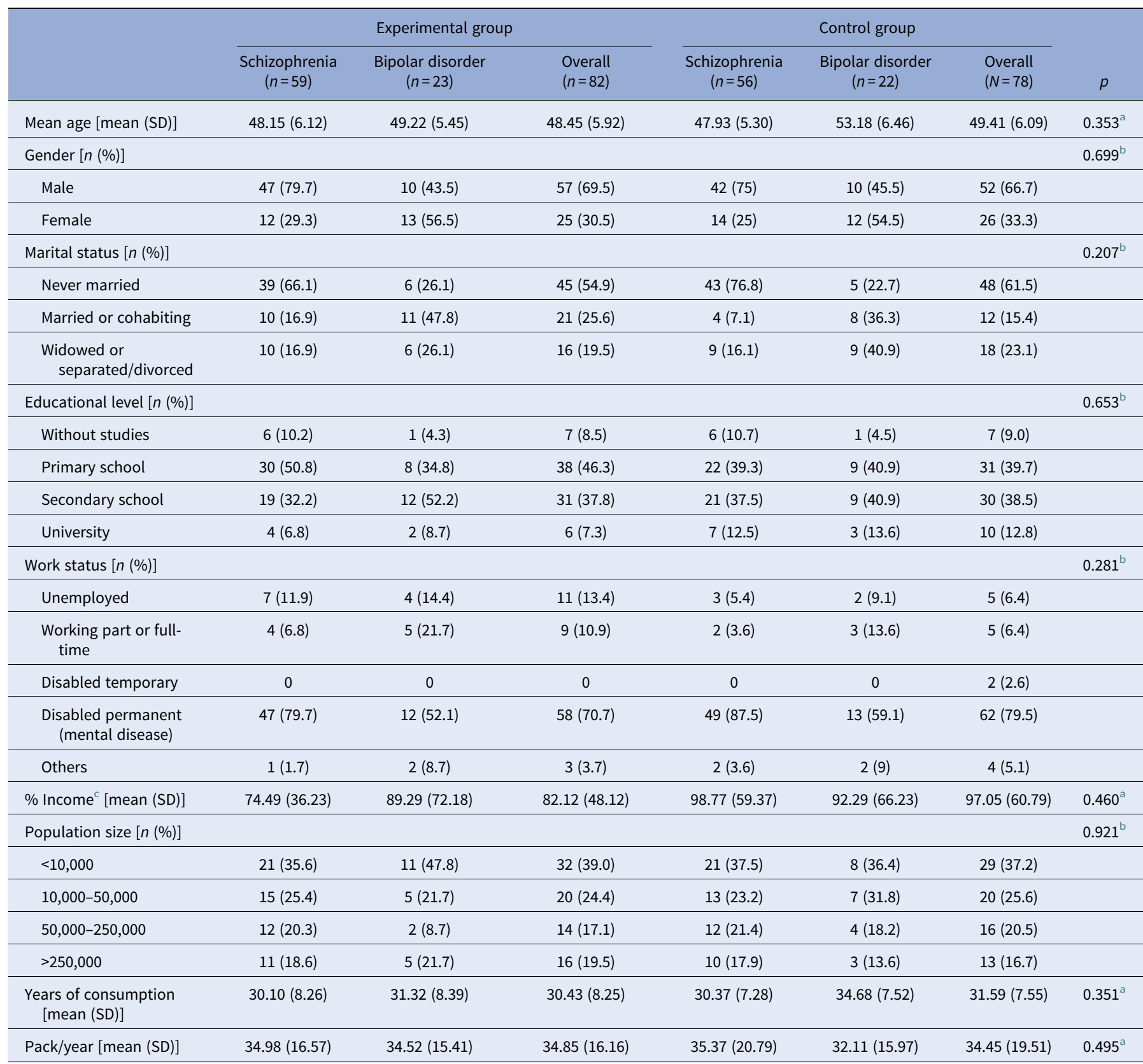


Table 1. Continued

\begin{tabular}{|c|c|c|c|c|c|c|c|}
\hline & \multicolumn{3}{|c|}{ Experimental group } & \multicolumn{3}{|c|}{ Control group } & \multirow[b]{2}{*}{$p$} \\
\hline & $\begin{array}{l}\text { Schizophrenia } \\
\quad(n=59)\end{array}$ & $\begin{array}{l}\text { Bipolar disorder } \\
\qquad(n=23)\end{array}$ & $\begin{array}{l}\text { Overall } \\
(n=82)\end{array}$ & $\begin{array}{l}\text { Schizophrenia } \\
\quad(n=56)\end{array}$ & $\begin{array}{l}\text { Bipolar disorder } \\
\qquad(n=22)\end{array}$ & $\begin{array}{l}\text { Overall } \\
(N=78)\end{array}$ & \\
\hline Expired $\mathrm{CO}^{\mathrm{d}}$ [mean (SD)] & $18.65(7.63)$ & $15.43(8.42)$ & $17.80(8.45)$ & 19.39 (9.09) & $17.36(6.34)$ & $16.93(9.00)$ & $0.433^{a}$ \\
\hline $\begin{array}{l}\text { Cigarettes per day [mean } \\
\text { (SD)] }\end{array}$ & $23.41(10.28)$ & $20.18(8.34)$ & $21.56(10.01)$ & $23.68(12.4)$ & $18.45(7.88)$ & $20.73(12.17)$ & $0.338^{a}$ \\
\hline FTND $^{e}$ [mean (SD)] & $5.91(2.01)$ & $5.39(2.31)$ & $5.76(2.09)$ & $5.92(2.29)$ & $5.41(2.26)$ & $5.77(2.28)$ & $0.886^{a}$ \\
\hline $\begin{array}{l}\text { Length of illness, years } \\
\text { [mean (SD)] }\end{array}$ & $23.76(8.05)$ & $21.56(10.14)$ & $23.15(8.68)$ & $25.18(7.78)$ & $21.90(9.19)$ & $24.25(8.27)$ & $0.439^{a}$ \\
\hline PANSS $^{f}$ total [mean (SD)] & $48.79(11.13)$ & - & $48.79(11.13)$ & $50.05(9.71)$ & - & $50.05(9.71)$ & $0.509^{a}$ \\
\hline $\mathrm{HDRS}^{\mathrm{g}}$ [mean (SD)] & - & $2.81(3.36)$ & $2.81(3.36)$ & - & $4.71(4.19)$ & $4.71(4.19)$ & $0.064^{a}$ \\
\hline YMRS $^{\text {h }}$ [mean (SD)] & - & $0.83(1.40)$ & $0.83(1.40)$ & - & $1.47(2.09)$ & $1.47(2.09)$ & $0.459^{a}$ \\
\hline HARS $^{i}$ [mean (SD)] & $5.49(5.10)$ & $4.78(4.50)$ & $5.29(4.92)$ & $6.13(5.70)$ & $8.25(8.96)$ & $6.70(6.74)$ & $0.264^{a}$ \\
\hline GAES [mean (SD)] & $65.17(13.23)$ & $75.00(12.34)$ & $67.96(13.66)$ & $63.46(11.57)$ & $72.38(9.30)$ & $65.90(11.65)$ & $0.366^{a}$ \\
\hline $\begin{array}{l}\text { Body Mass Index (BMI) } \\
\quad[\text { mean (SD)] }\end{array}$ & $30.27(5.36)$ & $31.22(6.02)$ & $30.54(5.53)$ & $30.10(5.92)$ & $31.08(6.31)$ & $30.38(6.01)$ & $0.893^{a}$ \\
\hline $\begin{array}{l}\text { Abdominal perimeter } \\
\text { [mean (SD)] }\end{array}$ & $107.42(13.97)$ & $106.23(15.41)$ & 107.09 (14.29) & $106.17(19.66)$ & $105.91(16.27)$ & $\begin{array}{l}106.10 \\
(18.66)\end{array}$ & $0.751^{a}$ \\
\hline $\begin{array}{l}\text { Alcohol consumption, Yes } \\
\quad[n(\%)]\end{array}$ & $17(28.8)$ & $2(8.7)$ & $19(23.2)$ & $5(8.9)$ & $5(23.8)$ & $10(12.8)$ & $0.233^{b}$ \\
\hline $\begin{array}{l}\text { Standard drink unit [mean } \\
\text { (SD)] }\end{array}$ & $5.94(8.03)$ & $4.00(1.41)$ & $5.74(7.60)$ & $6.20(6.14)$ & $13.00(6.40)$ & $9.60(6.92)$ & $0.115^{a}$ \\
\hline $\begin{array}{l}\text { History of Cannabis } \\
\text { consumption, Yes } \\
{[n(\%)]}\end{array}$ & $18(30.5)$ & 4 (17.4) & $22(26.8)$ & $18(32.1)$ & $7(31.8)$ & $25(32.1)$ & $0.469^{b}$ \\
\hline Joint/year [mean (SD)] & $20.69(35.84)$ & $83.30(95.86)$ & $32.08(54.43)$ & $10.76(14.23)$ & $20.44(21.89)$ & $13.71(17.02)$ & $0.982^{a}$ \\
\hline $\begin{array}{l}\text { Arterial hypertension, } \\
\quad \text { Yes }[n(\%)]\end{array}$ & $5(8.5)$ & $2(8.7)$ & $7(8.5)$ & $6(10.7)$ & $4(18.2)$ & $10(12.8)$ & $0.379^{b}$ \\
\hline $\begin{array}{l}\text { Diabetes mellitus, Yes } \\
\quad[n(\%)]\end{array}$ & 7 (11.9) & 4 (17.4) & $11(13.4)$ & $6(10.7)$ & $3(13.6)$ & $9(11.5)$ & $0.720^{b}$ \\
\hline Dislipemia, Yes [n (\%)] & $10(16.9)$ & $5(21.7)$ & $15(18.3)$ & $12(21.4)$ & $3(13.6)$ & $15(19.2)$ & $0.879^{b}$ \\
\hline $\begin{array}{l}\text { Evaluation of the SMS } \\
\text { [mean (SD)] }\end{array}$ & $75.31(18.01)$ & $70.56(19.70)$ & $73.92(18.46)$ & & & & \\
\hline
\end{tabular}

Abbreviations: BMI, Body Mass Index; SMS, short message service. ${ }^{a} U$ Mann-Whitney.

${ }^{\mathrm{b}} \mathrm{Chi}$-square test.

$c_{\%}$ income: \% respect for the interprofessional minimum salary in Spain (2017):707.06€.

${ }^{\mathrm{d}} \mathrm{CO}$ : expired carbon monoxide.

EFTND: Fagerström Test for Nicotine Dependence.

fPANSS: Positive and Negative Syndrome Scale.

${ }^{g}$ HDRS: Hamilton Rating Scale for Depression.

hYMRS: Young Mania Rating Scale.

'HARS: Hamilton Anxiety Rating Scale.

JGAES: Global Activity Evaluation Scale.

We decided to expose in the first place the efficacy of TIMER tool data and in the second place the results of lung damage because it can be better to understand globally the aims of the study.

\section{Efficacy}

\section{Abstinence}

Regarding the primary efficacy outcome in the long-term (week $48), 7.32 \%(n=6)$ of the EG and $3.85 \%(n=3)$ of the CG achieved abstinence at week 48 . At the short-term, $7.32 \%(n=6)$ of the EG and $2.56 \%(n=2)$ of the CG at week 12 , and $8.54 \%(n=7)$ of the EG and $5.13 \%(n=4)$ of the CG at week 24 , achieved abstinence. No statistically significant differences were observed between the groups at any point during the follow-up (Table 2).

\section{Tobacco reduction}

At week 12, both the TIMER and the 5A's intervention had generated a significant reduction in CPD (paired test, $p<0.01$ ) and expired CO (paired test, $p<0.01$ ).

These short-term significant reductions in expired $\mathrm{CO}$ and $\mathrm{CPD}$ were confirmed by a multivariate linear mixed-effects model (negative time effect, $p<0.001$ ) adjusted by other covariates which were added to the model by variable selection (see Table 3). 


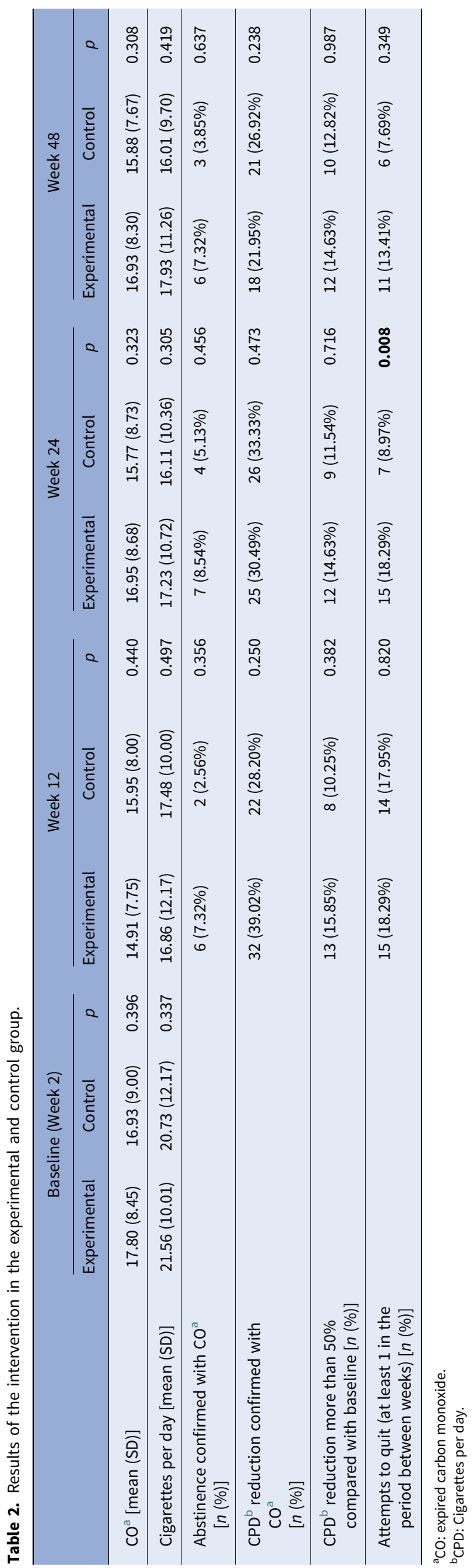

Mixed-effects models indicated that evolution in both $\mathrm{CO}$ levels and CPD was significant over time (time effect, $p<0.001$, Cohen's $d=-0.38$ ) and was also strongly predicted by the dependence level at baseline $(p<0.001$, Cohen's $d=-0.15)$. However, these significant reductions over time were not observed in the long-term multivariate model where was analyzed the evolution from baseline to 48 weeks.

At week 12, 32 patients (39.02\%) in the EG had reduced their CPD and expired CO, whereas 13 patients (15.85\%) achieved a reduction in at least $50 \% \mathrm{CPD}$. At week 24 , the combined reduction was observed in $25(30.49 \%)$ patients and a reduction in at least $50 \%$ CPD was achieved by 12 EG patients (14.63\%). At endpoint, 18 patients $(21.95 \%)$ had maintained a reduction in CPD and expired CO, whereas 12 patients (14.63\%) had a reduction in at least 50\% CPD. Significant differences from the CG in several measurements were not found at any visit (see Table 2 and Figure 2 for more details).

\section{Attempts to quit}

In the EG, $18.29 \%(n=15)$ of the patients made at least one attempt to abandon during the first 12 weeks of follow-up, without significant differences from the CG (see Table 2). Between weeks 12 and 24 , a significantly greater number of patients achieved this goal in the EG $(n=15 ; 18.29 \%)$ than in the CG $(n=7 ; 8.97 \%)(p=0.008)$. In the final observation period, between weeks 24 and $48,13.41 \%$ $(n=11)$ made at least one attempt in the EG, without significant differences from the CG.

\section{Spirometry results}

A total of 113 patients-mean age of 49.38 (5.99) consuming 36.61 (18.08) packs/year-completed the pulmonary function measurements with internal and external validation according to the scheduled protocol (see Jaen-Moreno 2019) [38]. Of these, $82 \mathrm{EG}$ patients were studied at the baseline visit and 31 CG patients wished to be informed about their lung function at the end of follow-up. The results of the spirometry tests are presented in Table 4.

A total of 27 patients (23.9\%) were diagnosed with COPD, regardless of psychiatric diagnoses (chi-square test, $p=0.176$ ), and sex (chi-square test, $p=0.163)$. Of these, six patients $(22.2 \%)$ were in the GOLD 1 stage, 20 (74.1\%) in GOLD 2 and 1 (3.7\%) in GOLD three stages. In the combined evaluation (presence of respiratory symptoms and lung function), 23 (85.2\%) patients met stage A criteria (low risk, low symptoms), 3 (11.1\%) patients stage B (low risk, more symptoms), and 1 (3.7\%) stage D (high risk, more symptoms) (see Table 4).

Univariate analysis with a logistic regression model (OR 1.77 CI 95\% 1,017-7.54 $p=0.046$ ) showed that living with other smokers increased the risk of COPD. None of the other analyzed variables showed any statistically significant associations.

Out of the 86 patients who did not meet COPD criteria, $23(26.7 \%)$ had an FEV1 $<80 \%$ than expected.

\section{Security protocol and satisfaction}

At study endpoint, EG patients rated the quality and convenience of the SMS as 73.92 (SD 18.46), out of 100 . Of the 82 subjects who took part in the intervention, 10 had no mobile phone, and these were called by a researcher using a landline. No subjects asked for the SMS or calls to be stopped, despite this being included as one of the options in the study's security protocol. 
Table 3. Linear mixed effects models to evaluate the changes in the reduction of tobacco over time (from baseline (Visit 0 ) to 12 weeks observations).

\begin{tabular}{|c|c|c|c|c|}
\hline & Coefficient & Standard error & $t$-Value & $p$ \\
\hline \multicolumn{5}{|l|}{ Model of $\mathrm{CO}^{\mathrm{a}}$} \\
\hline (Intercept) & 9.800 & 1.497 & 6.542 & $<0.001$ \\
\hline Time (baseline-12 weeks) & -0.031 & 0.006 & -4.729 & $<0.001$ \\
\hline FTND $^{b}$ & 1.466 & 0.238 & 6.141 & $<0.001$ \\
\hline \multicolumn{5}{|l|}{ Model of cigarettes per day } \\
\hline (Intercept) & 4.874 & $(1.781)$ & 2.735 & $<0.001$ \\
\hline Time (baseline-12 weeks) & -2.059 & $(0.377)$ & -5.455 & $<0.001$ \\
\hline FTND & 3.199 & $(0.271)$ & 11.797 & $<0.001$ \\
\hline
\end{tabular}

${ }^{\mathrm{a}} \mathrm{CO}$ : expired carbon monoxide.

bTND: Fagerström Test for Nicotine Dependence.

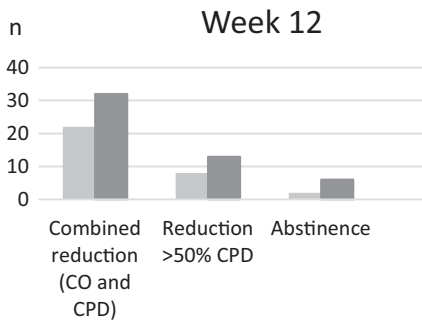

CO: expired carbon monoxide

CPD: cigarettes per day

$\mathrm{n}$ : number of patients

Figure 2. Effectiveness of the TIMER tool on smokers with an SMI.

\section{Discussion}

This pilot trial had two main objectives: to describe the prevalence of undetected COPD in a clinical sample of smokers with SMI, and to assess the value of the TIMER tool, which informs smokers of their respiratory risk and uses brief text messages to reinforce the intervention.

Regarding the lung damage goal, we found that in a sample of smokers with schizophrenia and $\mathrm{BD}$, spirometry enabled us to diagnose COPD_confirmed by post-bronchodilation values-in almost one in four patients studied $(23.9 \%)$, regardless of psychiatric disorder. In turn, the majority of them (78\%) presented moderate or severe stages (two and three of GOLD) and in most cases (85\%), COPD was evolving silently.

In relation with the efficacy outcome, the TIMER tool, which reports individual respiratory risks and the possibilities of prevention, promotes the detection of COPD in a sample of outpatients with SMI, generates attempts and significantly reduces consumption in the short term. Still, it does not show a significant difference with the control intervention and current reference tool in the clinical guidelines, in the primary goal of smoking cessation or any other outcome, in the long term.

\section{Lung damage}

So far, several surveys and population studies have reported a higher presence of COPD in individuals with schizophrenia (OR 1.57, 1.44-1.72) and BD (1.55, 1.45-1.67) [13]. However, the nature of this relationship remains uncertain [39]. To our knowledge, this is the first study on the presence of undiagnosed COPD by means of spirometry and its relationship with several risk variables for lung function in a representative sample of outpatients with schizophrenia and BD attending their scheduled appointments. Previous studies have explored this relationship in previously diagnosed patients obtained from a population survey [40]. In this case, they found a higher prevalence of COPD in both $\mathrm{BD}(\mathrm{OR}>2)$ and schizophrenia $(\mathrm{OR}>4)$, although COPD was significantly associated with smoking only in this last group.

COPD is a disease mainly associated with accumulated smoking and age but also influenced by living conditions [36]. The worldwide prevalence of COPD varies considerably [41] with an estimated $4 \%$ of COPD in smokers under 50 years of age [42]. Taken into account the age and tobacco consumption ( $>30$ packs/year) of our sample, the prevalence of COPD for the Spanish population is $9 \%$ [43]. In addition to the severity of smoking, lower educational level and poor living conditions may expose patients with an SMI to an increased risk of developing COPD.

Of the patients not diagnosed with COPD in the present study, $26.7 \%$ had an FEV1 lower than $80 \%$ of the expected rate. The lower FEV1 has been demonstrated to be a predictor of mortality due to cardiovascular, respiratory, and oncological causes, and it has been previously described in patients with SMI [44]. Moreover, FEV1 may explain at least in part an increased risk for a restrictive pattern (a decrease in FEV1 due to a lower FVC) and mainly related to central obesity and metabolic syndrome, which are common comorbidities in these patients $[45,46]$. 
Table 4. Respiratory damage and spirometrics results.

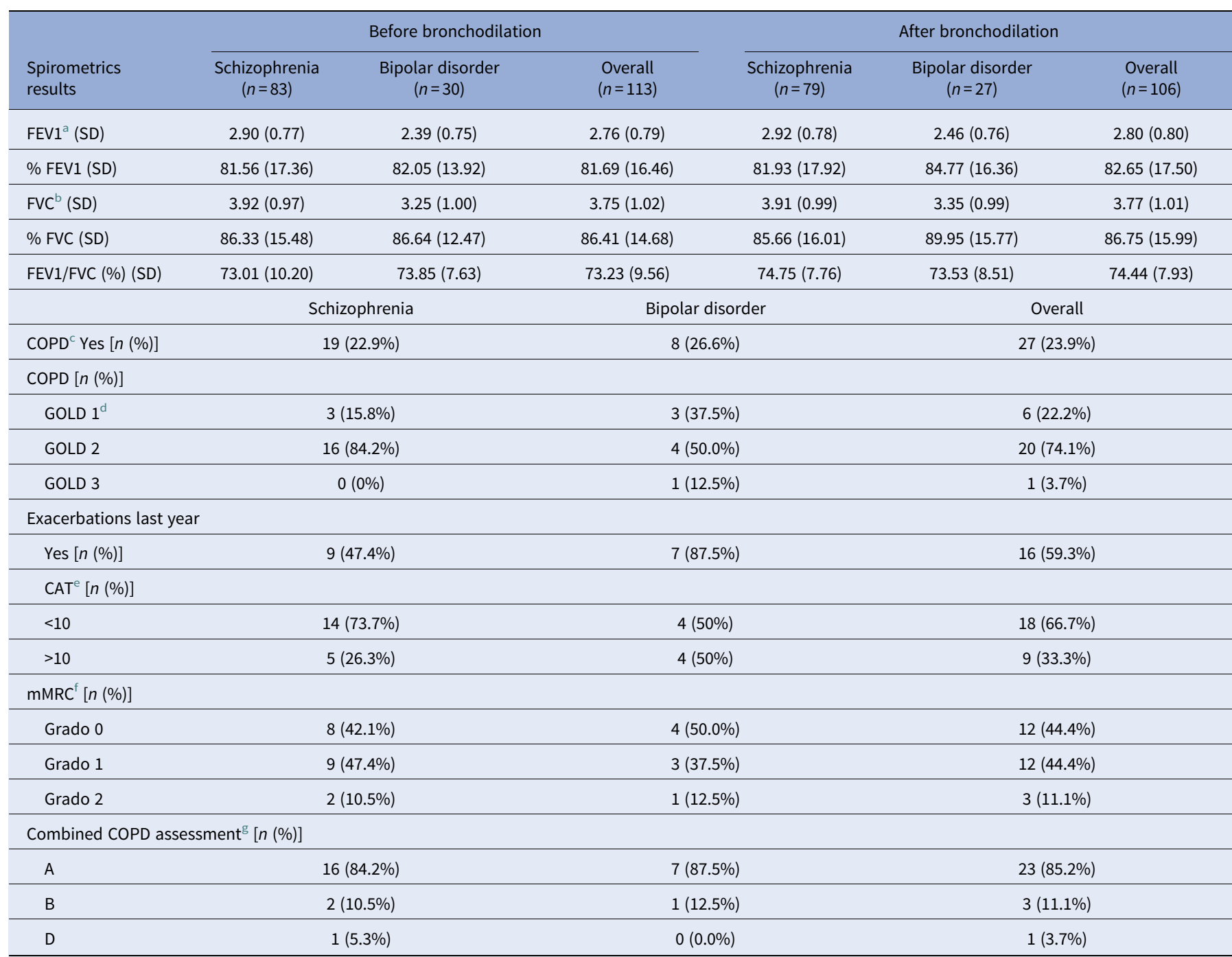

${ }^{\mathrm{a} F E V 1:}$ Forced expiratory volume in the first second;

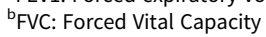

'COPD: chronic obstructive pulmonary disease

${ }^{\mathrm{d}}$ GOLD: Global Initiative for Chronic Obstructive Lung Disease

${ }^{\mathrm{e}} \mathrm{CAT}$ : COPD assessment test

${ }^{f}$ mMRC: Modified British Medical Research Council

${ }^{8}$ Combined COPD assessment: A (low risk, low symptoms): Gold 1-2, mMRC 0-1, CAT<10, exacerbations 0-1; B (low risk, more symptoms): Gold 1-2, mMRC > =2, CAT > = 10, exacerbations 0-1; C (high risk, less symptoms): Gold 3-4, mMRC $0-1$, CAT $<10$, exacerbations $>=2$; D (high risk, more symptoms): Gold 3-4, mMRC $>=2$, CAT $>=10$, exacerbations $>=2$.

Early detection of COPD in smokers with an SMI should be encouraged in order to increase their life expectancy. The co-existence of severe stages of COPD may worsen clinical outcomes in those with schizophrenia. For instance, a greater number of relapses leading to hospital admissions and a higher mortality rate in the 30 days after admission have been described [47]. Respiratory diseases-principally COPD and pneumonia - are the second cause of premature mortality in this population $[5,6]$. Indeed, patients with schizophrenia aged between 35 and 54 years have a risk of 11 times greater than that of the general population [48]. Moreover, having an obstructive pathology increases the development of comorbidities at an earlier age as well as the risk of premature mortality due to cardiovascular and oncological diseases [49].

\section{The TIMER intervention}

For the first time, smokers with SMI were offered the opportunity to learn about their lung damage. In this case as an opportunity to mobilize toward a change and evaluating the effect at the longitudinal level through a randomized pilot trial, controlled with an active comparator. The comparator chosen in this case was the $5 \mathrm{~A}$ 's intervention, which is the benchmark in clinical guidelines and far exceeds the current care strategies in MHCs in Southern Spain, which employ no more than $1 \mathrm{~A}[50]$.

The main goal of smoking cessation at week 48 and any other long-term outcomes, did not reach significant differences compared to the control intervention, 5 A's. In real-world clinical practice, when offered to all patients, without previous selection for their motivation level, a tool designed to generate initial mobilization without the guaranteed availability of pharmacological treatment, achieves up to $8.54 \%$ abstinence at week 24 and $7.32 \%$ by the end of follow-up. The goal of smoking cessation constitutes a complex challenge that is particularly difficult to maintain in this population. When pharmacological treatment was warranted to SMI patients who are actively seeking treatment, success rates at 6 
months were between 13 [14] and 15\% [51] and at 12 months were between 10 and $26 \%$ [14,52,53].

Reducing tobacco consumption may provide either a steppingstone to smoking cessation [54], or benefits to mitigate the damage [55]. TIMER, slowed only by the level of starting dependency, generated a significant reduction in the average levels of expired $\mathrm{CO}$ and decreased the tobacco consumption in nearly 4 in 10 patients, reported and objectified by expired CO. Half of these patients sustained this reduction until the end of the intervention, as well as 12 months after. Moreover, $15 \%$ of the patients at least halved the number of cigarettes smoked per day.

The TIMER tool used text messaging for the first 3 months. These types of messages have been shown to boost motivation in the general population and may increase the chances of success $[28,56]$. Indeed, in smoking treatment, a greater intensity of the intervention increases the cessation rates [57]. The design used in this study has not allowed differentiating separately the effect of the damage information and the technological element. Messages were rated as very satisfactory by patients, with none requesting them to be interrupted.

In the current pilot trial, no significant differences between interventions were observed during the follow-up. Although the 5 A's intervention has been scarcely studied in SMI, it was associated with abstinence rates (4\%), close to those described so far in this population: $6 \%$ abstinence at 12 months [58]. The two interventions have been limited by the lack of subsidies for pharmacological treatment: only two patients in each branch could access treatment. The option of pharmacological treatment after the motivational intervention was recommended to all patients. Even so, it is not part of the clinical routine of our environment, it was not available as a resource in the study and it was no publicly funded in Spain at that time. In smoking, initial motivation and perceived self-efficacy are predictors of initial progress (in a reduction of consumption and attempts to quit), but not of long-term success $[59,60]$. In the future, this type of intervention must be integrated into multi-component programs [15].

We should be cautious in interpreting the present results, which are based on a pilot trial with two main limitations. First, the reduced sample size may have limited the likelihood to detect a significant superiority of the motivational tool evaluated compared to the standard intervention. Second, premature mortality in this population is also linked to their economic vulnerability and although the TIMER tool was expected to mobilize the patient toward pharmacological treatment, the lack of available pharmacological resources in the study setting curbed this possibility.

Other limitations may have influenced the results of lung damage and efficacy of the intervention. A 22\% loss to follow-up was observed, which was similar for both study arms and falls under the usual rate in similar trials with this population [53]. The aim of adapting the trial to a real clinical context reduced the visits, thus limiting the opportunity for observation and not giving the approach the right specificity and intensity it deserves due to the severity of the addiction in SMI. Conversely, innovation and the randomized and controlled design are among the study strengths.

Further studies are needed to confirm the reported prevalence of undiagnosed COPD in clinical samples of patients with SMI. Although spirometry is not considered as a screening test in asymptomatic patients in the general population [36], the severity of smoking, the conditions and lifestyle habits in this population, their lack of preventive care [29] and the consequences of mortality in a late diagnosis could all point to the importance of studying lung function based on age ranges and accumulated tobacco use. Clinical trials with a larger sample size that guarantee a multicomponent approach will help to determine the real motivational value of tools that inform SMI patients of their chances of prevention.

To summarize, it was found that $24 \%$ of patients with SMI had previously undiagnosed COPD and that a motivational tool, which reports individual respiratory risks and is feasible in community care, may allow an early detection of lung damage.

However, although it generates quit attempts and reduces consumption in the short term, the TIMER tool did not significantly differ from the control intervention $5 \mathrm{~A}$ 's, in the goal of smoking cessation at 48 weeks or any other long-term outcome.

Acknowledgments. The authors would like to thank M. Osuna, M. Ayora, J. Caballero, P. Zurita, N. Novoa, J. Álvarez, J. Fernández, J. Redondo, M.S. López, I. Caro, F. Valdivia, C. Sádaba, R. Luque, and L. Padilla for their assistance. We also thank the altruistic and generous participation of all the patients in that project.

Funding. This study receives founding by the Spanish Ministry of Economy, Industry, and Competitiveness, Instituto Carlos III (FIS PI16/00802).

Conflict of interest. Vicent Balanzá-Martínez has received grants and served as consultant, advisor, or continuing medical education (CME) speaker during the last 5 years for the following entities: Angelini Spain, Angelini Portugal, Bristol-Myers-Squibb, Ferrer, Janssen, Juste, Lundbeck, Nutrición Médica, and Otsuka. He acknowledges the national grant PI16/01770 from the Instituto de Salud Carlos III, ISCIII (The PROBILIFE study)

Luis Gutiérrez-Rojas has been speaker for and advisory board member of Bristol-Myers Squibb, Janssen-Cilag, Astra-Zeneca, Rovi, Lundbeck, Otsuka, GSK, and Pfizer.

José Ángel Alcalá during the last 5 years, has received grants and served as consultant, advisor, or CME speaker for the following entities: Adamed,

Angelini, Casen Recordati, Janssen-Cilag, Lundbeck, Otsuka, Pfizer, and Servier.

Julio Bobes has received research grants and served as consultant, advisor, or speaker within de last 5 years for: AB-Biotics, Acadia Pharmaceuticals, Alkermes, Allergan, Ambrosseti-Angelini, Biogen, Casen Recordati, D\&A Pharma, Exeltis, Gilead, Indivior, GW Pharmaceuticals, Janssen-Cilag, Jazz Pharmaceuticals, Lundbeck, Mundipharma, Newron, Otsuka, Pfizer, Roche, Sage Therapeutics, Servier, Schwabe Farma Ibérica, Shire, Takeda, research funding from the Spanish Ministry of Economy and Competitiveness-Centro de Investigación Biomedica en Red area de Salud Mental (CIBERSAM) and Instituto de Salud Carlos III-, Spanish Ministry of Health, Social Services and Equality-Plan Nacional sobre Drogas- and the 7th Framework Program of the European Union.

All other researchers report no biomedical financial interests or potential conflicts of interests.

Data availability statement. The data that support the findings of this study are available from the corresponding author upon reasonable request.

\section{References}

[1] Chesney E, Goodwind GM, Fazel S. Risks of all-cause and suicide mortality in mental disorders: a meta-review. World Psychiatry. 2014;13:153-60.

[2] Walker ER, McGee RE, Druss BG. Mortality in mental disorders and global disease burden implications: a systematic review and meta-analysis. JAMA Psychiatry. 2015;72:334-41. doi: 10.1001/jamapsychiatry.2014.2502.

[3] Hjorthøj C, Stürup AE, McGrath JJ, Nordentoft M. Years of potential life lost and life expectancy in schizophrenia: a systematic review and metaanalysis. Lancet Psychiatry. 2017;4:295-301. doi: 10.1016/S2215-0366(17) 30078-0.

[4] De Hert M, Correll CU, Bobes J, Cetkovich-Bakmas M, Cohen DAN, Asai I, et al. Physical illness in patients with severe mental disorders. 
I. Prevalence, impact of medications and disparities in health care. World Psychiatry. 2011;10:52-77. doi: 10.1002/j.2051-5545.2011.tb00014.x.

[5] Crump C, Sundquist K, Winkleby MA, Sundquist J. Comorbidities and mortality in bipolar disorder: a Swedish national cohort study. JAMA Psychiatry. 2013;70:931-9. doi: 10.1001/jamapsychiatry.2013.1394.

[6] Olfson M, Gerhard T, Huang C, Crystal S, Stroup TS. Premature mortality among adults with schizophrenia in the United States. JAMA Psychiatry. 2015;72:1172-81. doi: 10.1001/jamapsychiatry.2015.1737.

[7] Hayes JF, Miles J, Walters K, King M, Osborn DP. A systematic review and meta-analysis of premature mortality in bipolar affective disorder. Acta Psychiatr Scand. 2015;131:417-25. doi: 10.1111/acps.12408.

[8] Schroeder SA. Even more illness caused by smoking than previously estimated. JAMA Intern Med. 2014;174:1928-9. doi: 10.1001/jamainternmed.2014.4297.

[9] Dickerson F, Adamos MB, Katsafanas E, Khushalani S, Origoni A, Savage CLG, et al. The association among smoking, HSV-1 exposure, and cognitive functioning in schizophrenia, bipolar disorder, and non-psychiatric controls. Schizophr Res. 2016;176:566-71. doi: 10.1016/j. schres.2016.05.022.

[10] Diaz FJ, James D, Botts S, Maw L, Susce MT, de Leon J. Tobacco smoking behaviors in bipolar disorder: a comparison of the general population, schizophrenia, and major depression. Bipolar Disord. 2009;11:154-65. doi: 10.1111/j.1399-5618.2009.00664.x.

[11] de Leon J, Diaz FJ. Genetics of schizophrenia and smoking: an approach to studying their comorbidity based on epidemiological findings. Hum Genet. 2012;131:877-901. doi: 10.1007/s00439-011-1122-6.

[12] Lê Cook B, Wayne GF, Kafali EN, Liu Z, Shu C, Flores M. Trends in smoking among adults with mental illness and association between mental health treatment and smoking cessation. JAMA-J Am Med Assoc. 2014; 311:172-82. doi: 10.1001/jama.2013.284985.

[13] Zareifopoulos N, Bellou A, Spiropoulou A, Spiropoulos K. Prevalence of comorbid chronic obstructive pulmonary disease in individuals suffering from schizophrenia and bipolar disorder: a systematic review. COPD. 2018;15:612-20. doi: 10.1080/15412555.2019.1572730.

[14] Anthenelli RM, Benowitz NL, West R, St Aubin L, McRae T, Lawrence D, et al. Neuropsychiatric safety and efficacy of varenicline, bupropion, and nicotine patch in smokers with and without psychiatric disorders (EAGLES): a double-blind, randomised, placebo-controlled clinical trial. Lancet. 2016;387:2507-20. doi: 10.1016/s0140-6736(16)30272-0.

[15] Garcia-Portilla MP, Garcia-Alvarez L, Sarramea F, Galvan G, Diaz-Mesa E, Bobes-Bascaran T, et al. It is feasible and effective to help patients with severe mental disorders to quit smoking: an ecological pragmatic clinical trial with transdermal nicotine patches and varenicline. Schizophr Res. 2016;176:272-80. doi: 10.1016/j.schres.2016.05.011.

[16] Stubbs B, Vancampfort D, Bobes J, De Hert M, Mitchell AJ. How can we promote smoking cessation in people with schizophrenia in practice? A clinical overview. Acta Psychiatr Scand. 2015;132:122-30. doi: 10.1111/ acps.12412.

[17] Sarramea Crespo F, Jaén-Moreno MJ, Gutiérrez-Rojas L, Balanzá-Martínez V, García-Alvarez L, Saiz Martínez PA, et al. "Readiness to change" predicts efficacy of reduction among smokers with severe mental illness. Eur Addict Res. 2019;25:256-62. doi: 10.1159/000500450.

[18] Gilbody S, Peckham E, Bailey D, Arundel C, Heron P, Crosland S, et al. Smoking cessation for people with severe mental illness (SCIMITAR+): a pragmatic randomised controlled trial. Lancet Psychiatry. 2019;6:379-90. doi: 10.1016/S2215-0366(19)30047-1.

[19] Balanzá-Martínez V, Atienza-Carbonell B, Kapczinski F, De Boni RB. Lifestyle behaviours during the COVID-19-time to connect. Acta Psychiatr Scand. 2020;141:399-400. doi: 10.1111/acps.13177.

[20] Górecka D, Bednarek M, Nowiński A, Puścińska E, Goljan-Geremek A, Zieliński J. Diagnosis of airflow limitation combined with smoking cessation advice increases stop-smoking rate. Chest. 2003;123:1916-23. doi: 10.1378/chest.123.6.1916.

[21] Bednarek M, Gorecka D, Wielgomas J, Czajkowska-Malinowska M, Regula J, Mieszko-Filipczyk G, et al. Smokers with airway obstruction are more likely to quit smoking. Thorax. 2006;61:869-73. doi: 10.1136/ thx.2006.059071.
[22] Parkes G, Greenhalgh T, Griffin M, Dent R. Effect on smoking quit rate of telling patients their lung age: the Step2Quit randomised controlled trial. BMI. 2008;336:598-600. doi: 10.1136/bmj.39503.582396.25.

[23] Young RP, Hopkins RJ, Smith M, Hogarth DK. Smoking cessation: the potential role of risk assessment tools as motivational triggers. Postgrad Med J. 2010;86:22-6. doi: 10.1136/pgmj.2009.084947.

[24] Kaminsky DA, Marcy T, Dorwaldt A, Pinckney R, DeSarno M, Solomon L, et al. Motivating smokers in the hospital pulmonary function laboratory to quit smoking by use of the lung age concept. Nicotine Tob Res Off J Soc Res Nicotine Tob. 2011;13:1161-6. doi: 10.1093/ntr/ntr096.

[25] Drummond MB, Astemborski J, Lambert AA, Goldberg S, Stitzer ML, Merlo CA, et al. A randomized study of contingency management and spirometric lung age for motivating smoking cessation among injection drug users. BMC Publ Health. 2014;14:761. doi: 10.1186/1471-2458-14761.

[26] Kelly DL, Raley HG, Lo S, Wright K, Liu F, McMahon RP, et al. Perception of smoking risks and motivation to quit among nontreatment-seeking smokers with and without schizophrenia. Schizophr Bull. 2012;38:543-51. doi: $10.1093 /$ schbul/sbq124.

[27] Thomson D, Berk M, Dodd S, Rapado-Castro M, Quirk SE, Ellegaard PK, et al. Tobacco use in bipolar disorder. Clin Psychopharmacol Neurosci. 2015;13:1-11. doi: 10.9758/cpn.2015.13.1.1.

[28] Whittaker R, McRobbie H, Bullen C, Rodgers A, Gu Y. Mobile phonebased interventions for smoking cessation. Cochrane Database Syst Rev. 2016;4:CD006611. doi: 10.1002/14651858.CD006611.pub4.

[29] Liu NH, Daumit GL, Dua T, Aquila R, Charlson F, Cuijpers P, et al. Excess mortality in persons with severe mental disorders: a multilevel intervention framework and priorities for clinical practice, policy and research agendas. World Psychiatry. 2017;16:30-40. doi: 10.1002/wps.20384.

[30] Vestbo J, Hurd SS, Agustí AG, Jones PW, Vogelmeier C, Anzueto A, et al. Global strategy for the diagnosis, management, and prevention of chronic obstructive pulmonary disease. Am J Respir Crit Care Med. 2013;187: 347-65. doi: 10.1164/rccm.201204-0596PP.

[31] First M, Spitzer RL, Gibbon M, Williams JBW. Entrevista clínica estructurada para los trastornos del eje I del DSM-IV: SCID-I. Versión Clínica Ed Masson Barcelona. 1999.

[32] Bobes J, Bulbena A, Luque A, Dal-Re R, Ballesteros J, Ibarra N. A comparative psychometric study of the Spanish versions with 6,17, and 21 items of the Hamilton Depression Rating Scale. Med Clin (Barc). 2003; 120:693-700.

[33] Colom F, Vieta E, Martinez-Aran A, Garcia-Garcia M, Reinares M, Torrent C, et al. Spanish version of a scale for the assessment of mania: validity and reliability of the Young Mania Rating Scale. Med Clin (Barc). 2002;119:366-71.

[34] Peralta V, Cuesta MJ. Psychometric properties of the positive and negative syndrome scale (PANSS) in schizophrenia. Psychiatry Res. 1994;53:31-40. doi: 10.1016/0165-1781(94)90093-0.

[35] Harris PA, Taylor R, Thielke R, Payne J, Gonzalez N, Conde JG. Research electronic data capture (REDCap) - a metadata-driven methodology and workflow process for providing translational research informatics support. J Biomed Inform. 2009;42:377-81. doi: 10.1016/j.jbi.2008.08.010.

[36] Vogelmeier CF, Criner GJ, Martinez FJ, Anzueto A, Barnes PJ, Bourbeau J, et al. Global strategy for the diagnosis, management, and prevention of chronic obstructive lung disease 2017 report. GOLD executive summary. Am J Respir Crit Care Med. 2017;195:557-82. doi: 10.1164/rccm.2017010218P.

[37] Fletcher C, Peto R. The natural history of chronic airflow obstruction. Br Med J. 1977;1:1645-8.

[38] Jaén-Moreno MJ, Feu N, Redondo-Écija J, Montiel FJ, Gómez C, del Pozo GI, et al. Smoking cessation opportunities in severe mental illness (tobacco intensive motivational and estimate risk-TIMER-): study protocol for a randomized controlled trial. Trials. 2019;20:47. doi: 10.1186/s13063-0183139-9.

[39] Krieger I, Tzur Bitan D, Comaneshter D, Cohen A, Feingold D. Increased risk of smoking-related illnesses in schizophrenia patients: a nationwide cohort study. Schizophr Res. 2019. doi: 10.1016/j.schres.2019.07.058.

[40] Partti K, Vasankari T, Kanervisto M, Perala J, Saarni SI, Jousilahti P, et al. Lung function and respiratory diseases in people with psychosis: 
population-based study. Br J Psychiatry. 2015;207:37-45. doi: 10.1192/bjp. bp.113.141937.

[41] Barrecheguren M, González C, Miravitlles M. What have we learned from observational studies and clinical trials of mild to moderate COPD? Respir Res. 2018;19:177. doi: 10.1186/s12931-018-0882-0.

[42] Soriano JB, Polverino F, Cosio BG. What is early COPD and why is it important? Eur Respir J. 2018;52. doi: 10.1183/13993003.01448-2018.

[43] Miravitlles M, Soriano JB, García-Río F, Muñoz L, Duran-Tauleria E, Sanchez G, et al. Prevalence of COPD in Spain: impact of undiagnosed COPD on quality of life and daily life activities. Thorax. 2009;64:863-8. doi: 10.1136/thx.2009.115725.

[44] Filik R, Sipos A, Kehoe PG, Burns T, Cooper SJ, Stevens H, et al. The cardiovascular and respiratory health of people with schizophrenia. Acta Psychiatr Scand. 2006;113:298-305. doi: 10.1111/j.16000447.2006.00768.x.

[45] Vancampfort D, Probst M, Stubbs B, Soundy A, De Herdt A, De Hert M. Metabolic syndrome and lung function in schizophrenia: a pilot study. Psychiatry Res. 2014;220:58-62. doi: 10.1016/j.psychres.2014.06.008.

[46] Vancampfort D, Probst M, Stubbs B, Soundy A, De Herdt A, De Hert M. Associations between expiratory spirometry parameters and limitations in daily life activities in patients with schizophrenia. Gen Hosp Psychiatry. 2014;36:172-6. doi: 10.1016/j.genhosppsych.2013.11.001.

[47] Jørgensen M, Mainz J, Lange P, Johnsen SP. Quality of care and clinical outcomes of chronic obstructive pulmonary disease in patients with schizophrenia. A Danish nationwide study. Int J Qual Heal Care. 2018; 30:351-7. doi: 10.1093/intghc/mzy014.

[48] Hayes JF, Marston L, Walters K, King MB, Osborn DPJ. Mortality gap for people with bipolar disorder and schizophrenia: UK-based cohort study 2000-2014. Br J Psychiatry. 2017;211:175-81. doi: 10.1192/bjp. bp.117.202606.

[49] Divo MJ, Celli BR, Poblador-Plou B, Calderón-Larrañaga A, de-Torres JP, Gimeno-Feliu LA, et al. Chronic Obstructive Pulmonary Disease (COPD) as a disease of early aging: evidence from the EpiChron Cohort. PLoS One. 2018;13:e0193143. doi: 10.1371/journal.pone.0193143.

[50] Sarramea F, Jaen-Moreno MJ, Balanzá-Martínez V, Osuna MI, Alcalá JÁ, Montiel FJ, et al. Setting the stage to quit smoking in bipolar disorder patients: brief advice in clinical practice. Adicciones. 2019;31:136-45. doi: 10.20882/adicciones.1006.

[51] Williams JM, Steinberg ML, Zimmermann MH, Gandhi KK, Stipelman B, Budsock PD, et al. Comparison of two intensities of tobacco dependence counseling in schizophrenia and schizoaffective disorder. J Subst Abus Treat. 2010;38:384-93. doi: 10.1016/j.jsat.2010.03.006.

[52] Peckham E, Brabyn S, Cook L, Tew G, Gilbody S. Smoking cessation in severe mental ill health: what works? an updated systematic review and meta-analysis. BMC Psychiatry. 2017;17:252. doi: 10.1186/s12888-0171419-7.

[53] Gilbody S, Peckham E, Man MS, Mitchell N, Li J, Becque T, et al. Bespoke smoking cessation for people with severe mental ill health (SCIMITAR): a pilot randomised controlled trial. Lancet Psychiatry. 2015;2:395-402. doi: 10.1016/s2215-0366(15)00091-7.

[54] Cinciripini PM, Lapitsky L, Seay S, Wallfisch A, Kitchens K, Van Vunakis $\mathrm{H}$. The effects of smoking schedules on cessation outcome: can we improve on common methods of gradual and abrupt nicotine withdrawal? J Consult Clin Psychol. 1995;63:388-99. doi: 10.1037//0022-006x.63.3.388.

[55] Kunze M. Maximizing help for dissonant smokers. Addiction. 2000;95 Suppl 1:S13-S13827. doi: 10.1111/add.13685.

[57] Fiore MC, Jaén CR, Baker TB, Bailey WC, Benowitz NL, Curry SJ, et al. Treating tobacco use and dependence: 2008 update. Rockville, MD US Dep Heal Hum Serv; 2008.

[58] DiClemente CC, Delahanty JC, Kofeldt MG, Dixon L, Goldberg R, Lucksted A. Stage movement following a 5A's intervention in tobacco dependent individuals with serious mental illness (SMI). Addict Behav. 2011;36:261-4. doi: 10.1016/j.addbeh.2010.11.002.

[59] Borland R, Yong HH, Balmford J, Cooper J, Cummings KM, O'Connor RJ, et al. Motivational factors predict quit attempts but not maintenance of smoking cessation: findings from the International Tobacco Control Four country project. Nicotine Tob Res. 2010;12(Suppl):S4-11. doi: 10.1093/ ntr/ntq050.

[60] Lee HS, Catley D, Harris KJ. Improving understanding of the quitting process: psychological predictors of quit attempts versus smoking cessation maintenance among college students. Subst Use Misuse. 2014;49: 1332-9. doi: 10.3109/10826084.2014.901386. 